\title{
First submicroscopic inversion of the OPA1 gene identified in dominant optic atrophy - a case report
}

\author{
Nicole Weisschuh $^{1 *+}$ (D), Pascale Mazzola ${ }^{2 \dagger}$, Tilman Heinrich ${ }^{2,3}$, Tobias Haack ${ }^{2,3}$, Bernd Wissinger ${ }^{1}$, Felix Tonagel ${ }^{4}$ and \\ Carina Kelbsch ${ }^{4}$
}

\begin{abstract}
Background: Dominant optic atrophy (DOA) is an inherited optic neuropathy that mainly affects visual acuity, central visual fields and color vision due to a progressive loss of retinal ganglion cells and their axons that form the optic nerve. Approximately 45-90\% of affected individuals with DOA harbor pathogenic variants in the OPA1 gene. The mutation spectrum of OPA1 comprises nonsense, canonical and non-canonical splice site, frameshift and missense as well as copy number variants, but intragenic inversions have not been reported so far.

Case presentation: We report a 33-year-old male with characteristic clinical features of DOA. Whole-genome sequencing identified a structural variant of $2.4 \mathrm{~kb}$ comprising an inversion of $937 \mathrm{bp}$ at the OPA 1 locus. Fine mapping of the breakpoints to single nucleotide level revealed that the structural variation was an inversion flanked by two deletions. As this rearrangement inverts the entire first exon of OPA1, it was classified as likely pathogenic.
\end{abstract}

Conclusions: We report the first DOA case harboring an inversion in the OPA1 gene. Our study demonstrates that copy-neutral genomic rearrangements have to be considered as a possible cause of disease in DOA cases.

Keywords: Case report, Inversion, Complex rearrangement, Dominant optic atrophy, OPA1, Non-homologous end joining (NHEJ)

\section{Background}

Dominant optic atrophy (DOA, MIM\#165500) and Leber's hereditary optic neuropathy (LHON, MIM\#535000) are the two most common entities of inherited optic neuropathies seen in clinical practice [1]. DOA was first described in the 1950 s and is genetically and clinically distinct from LHON $[2,3]$. Clinically, affected individuals with DOA present with temporally accented pallor of the optic nerve head at fundus examination, and bilateral central or caeco-central scotoma at visual field examination. A laterally symmetrical temporal reduced thickness of the retinal nerve fiber layer

\footnotetext{
* Correspondence: nicole.weisschuh@uni-tuebingen.de

${ }^{\dagger}$ Nicole Weisschuh and Pascale Mazzola contributed equally to this work. ${ }^{1}$ Institute for Ophthalmic Research, Centre for Ophthalmology, University of Tübingen, Tübingen, Germany

Full list of author information is available at the end of the article
}

(RNFL) is seen upon optical coherence tomography imaging (OCT). Other than in affected individuals diagnosed with LHON, who often show red-green dyschromatopsia, the color vision defect in DOA reflects a generalized dyschromatopsia or is specific to the tritan axis [4]. Visual acuity can range from 20/20 to light perception, with $40 \%$ of affected individuals having a visual acuity over 20/60 [5].

Depending on the population studied, $45-90 \%$ of DOA cases harbor pathogenic variants in $O P A 1[6,7]$, which was the first gene to be described as an underlying cause of DOA $[8,9]$. OPA1 encodes a dynamin-related GTPase which is imported into mitochondria and plays an important role in mitochondrial dynamics and structural maintenance of the cristae junctions [10, 11]. As of August 2020, the Human Gene Mutation Database (HGMD) lists

C C The Author(s). 2020 Open Access This article is licensed under a Creative Commons Attribution 4.0 International License, which permits use, sharing, adaptation, distribution and reproduction in any medium or format, as long as you give appropriate credit to the original author(s) and the source, provide a link to the Creative Commons licence, and indicate if changes were made. The images or other third party material in this article are included in the article's Creative Commons licence, unless indicated otherwise in a credit line to the material. If material is not included in the article's Creative Commons licence and your intended use is not permitted by statutory regulation or exceeds the permitted use, you will need to obtain permission directly from the copyright holder. To view a copy of this licence, visit http://creativecommons.org/licenses/by/4.0/ The Creative Commons Public Domain Dedication waiver (http://creativecommons.org/publicdomain/zero/1.0/) applies to the data made available in this article, unless otherwise stated in a credit line to the data. 
404 disease-causing variants in OPA1. Most variants are private and only few constitute founder alleles [7, 12]. Overall, the majority of disease-causing variants are loss-of-function alleles, indicating that haploinsufficiency is the predominant disease mechanism underlying OPA1-linked DOA [12, 13]. The mutation spectrum comprises nonsense, canonical splice site, frameshift and missense variants. In addition, copy number variants $(\mathrm{CNVs})$ and deep-intronic variants inducing aberrant splicing have been reported [14-17], necessitating a comprehensive genetic diagnostic testing including the analysis of read depth and intronic sequences. So far, submicroscopic inversions in the OPA1 gene have not yet been reported as a cause of DOA. Since inversions are copy-neutral, they escape detection by conventional diagnostic technologies such as microarrays and read depth methods. We here present the first affected individual with DOA in whom whole genome sequencing based on short read technology led to the identification of a complex structural rearrangement including the first coding exon of OPA1. Our findings demonstrate that copy-neutral genomic rearrangements have to be considered as a possible cause of disease in DOA.

\section{Case presentation}

The 33-year-old male subject of German descent presented with a history of bilateral slowly progressive vision loss. His younger brother was reported to have "bad vision" but was not available for phenotypic and genetic analysis. Parents and grandparents were apparently unaffected. Cerebral imaging (magnetic resonance imaging with contrast) had not revealed any underlying cause, particularly no spaceoccupying lesions compromising the visual pathway.

\section{Clinical investigation}

At examination, best-corrected visual acuity was 0.5 (right eye) and 0.4 (left eye). According to previous eye examinations, visual acuity was documented to have been 0.8 in both eyes 10 years ago with a continuous slowly progressive decline.

Anterior segment slit-lamp examination was unremarkable. Intraocular eye pressure was within normal limits, and no relative afferent pupillary defect was found. Fundus ophthalmoscopy unveiled bilateral symmetric, temporally accented optic disc pallor, which is a typical and characteristic finding of DOA (Fig. 1). Correspondingly, the temporal optic atrophy could be confirmed by bilaterally reduced RNFL in OCT (Fig. 2).

Visual field examination of the central $30^{\circ}$ of the visual field was relatively unremarkable with only slight paracentral relative defects $\mathrm{R}>\mathrm{L}$ (Additional file 1). Color testing with Panel-D15 was significant for a dyschromatopsia to the tritan axis $\mathrm{L}>\mathrm{R}$ (Additional file 2).

In summary, the affected individual presented with characteristic clinical findings consistent with a diagnosis of DOA.

\section{Genetic analysis}

Genomic DNA was extracted from peripheral blood using standard protocols. Whole genome sequencing $(2 \times 150 \mathrm{bp}$ paired-end reads) was performed on an Illumina platform (NovaSeq6000). The average coverage on target was 54.8x $(99.76 \%>20 x)$. Bioinformatic processing of raw read data, annotation and variant calling was performed as described previously [18]. For details refer to the megSAP pipeline (https://github.com/imgag/megSAP) developed at the Institute of Medical Genetics and Applied Genomics, University Hospital Tübingen, Germany. Bioinformatic analysis and genomic coordinates given in this manuscript are based on the GRCh37 genome (hg19). The Manta Structural Variant Caller [19] identified $\mathrm{a} \sim 1 \mathrm{kB}$ spanning inversion that changes the orientation of the first coding exon of OPA1. Manual inspection of split reads using the Integrative Genomics Viewer (IGV, version 2.3, see Fig. 3) revealed a complex rearrangement. Subsequent breakpoint PCR and Sanger sequencing was performed in order to resolve the variant configuration to single nucleotide resolution.
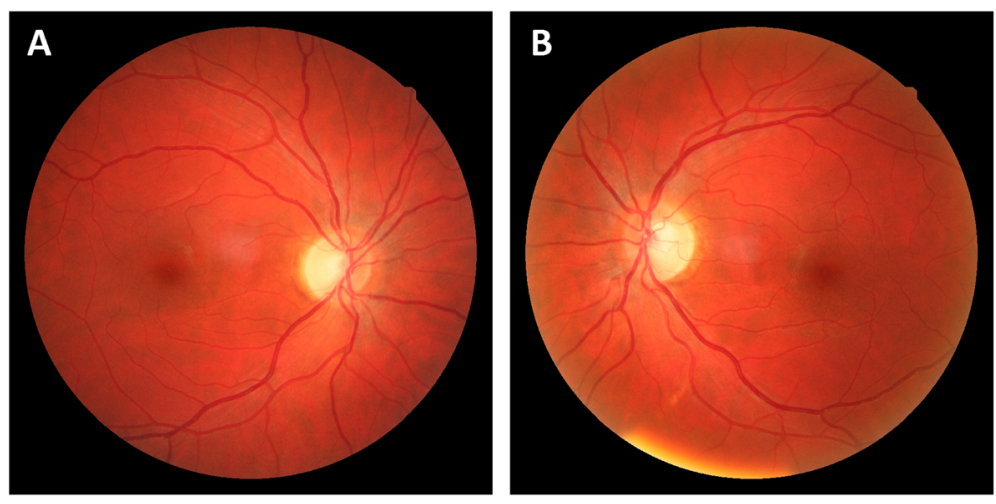

Fig. 1 Eye fundi. Temporal pallor of discs is seen in the right eye (a) and the left eye (b) of the affected proband 

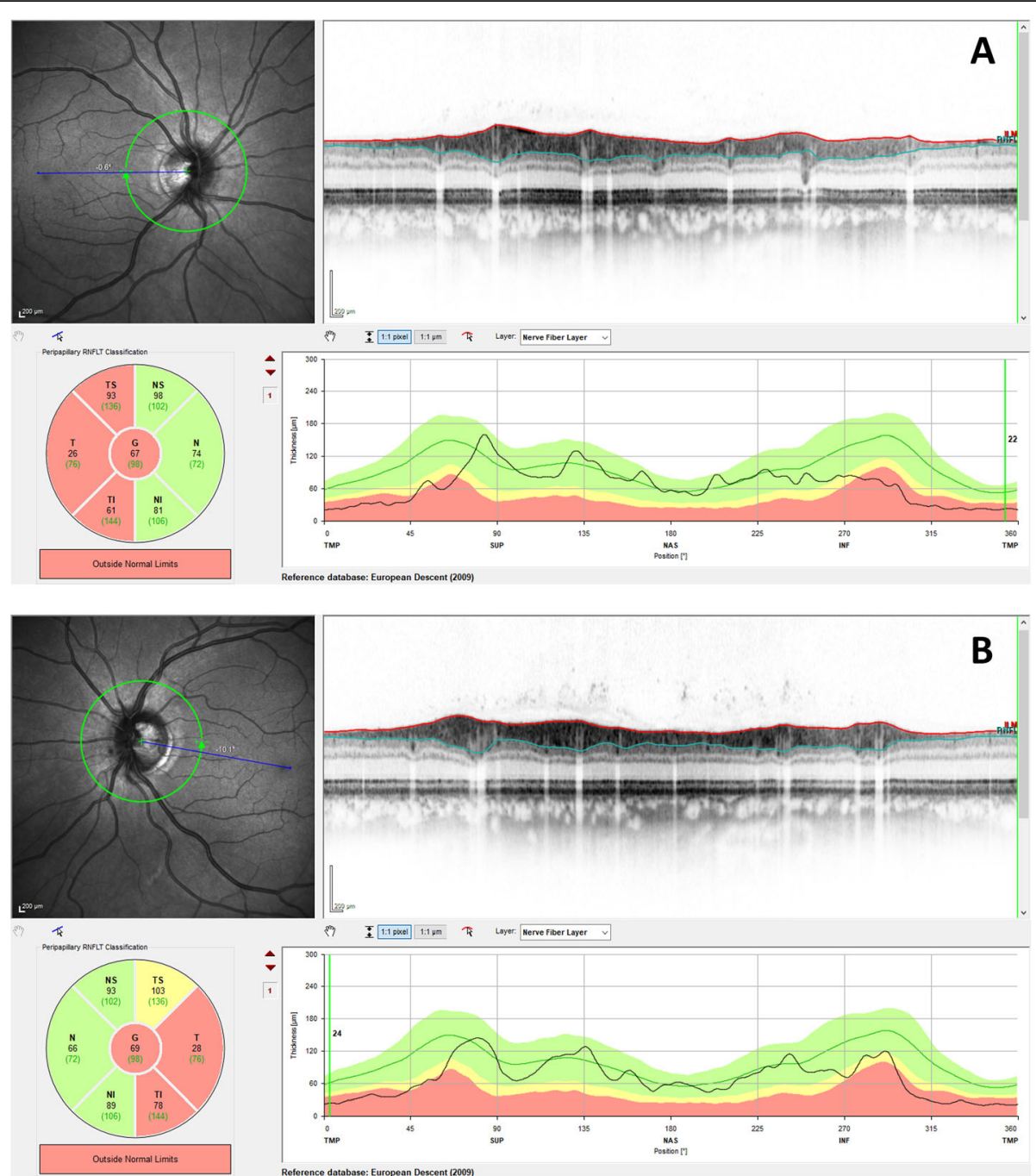

Fig. 2 Optical coherence tomography (OCT) of the retinal nerve fiber layers (RNFLs). The right eye (a) and left eye (b) OCT of the affected proband show temporal thinning of RNFLs

Figure 4a gives a schematic overview of the structural variant, and Fig. 4b shows the Sanger sequencing of the breakpoint junctions. The nomenclature for the variant according to the guidelines established by the Human Genome Variation Society (HGVS) [20] was established as follows: NC_000003.11:g.193,310,511_193,312,932delins193, 310,605_193,311,825[193,310,605_193,311,541inv].

It was not possible to obtain an RNA sample from the proband, excluding transcript analysis. However, since the inversion comprises the first coding exon of the OPA1 gene including the translational start site, it most likely constitutes a loss-of-function allele.

\section{Discussion and conclusions}

This is the first report of a submicroscopic inversion involving the OPA1 gene. Inversions are mainly mediated by three mechanisms: While non-allelic homologous recombination
(NAHR) and the fork stalling and template switching (FoSTeS) mechanism require homologous sequences at the breakpoints, non-homologous end joining (NHEJ) is a repair mechanism for double-strand breaks [21-24]. In the latter, the inverted sequence directly ligates to the breakpoint, independent of sequence homology. Using the online tools Blast 2 Sequences (http://polyp.biochem. uci.edu/blast/wblast2.html) and Repeat Masker (http:// www.repeatmasker.org), we analyzed the sequences flanking the breakpoints for similarities to each other and to repeat elements that can mediate genomic rearrangements (data not shown). The lack of sequence homology at the breakpoints and the sequencing results of breakpoint junctions indicate that the structural variant described in this study most likely involved multiple double-strand breaks and the rejoining of DNA fragments by NHEJ. Of note, we followed 


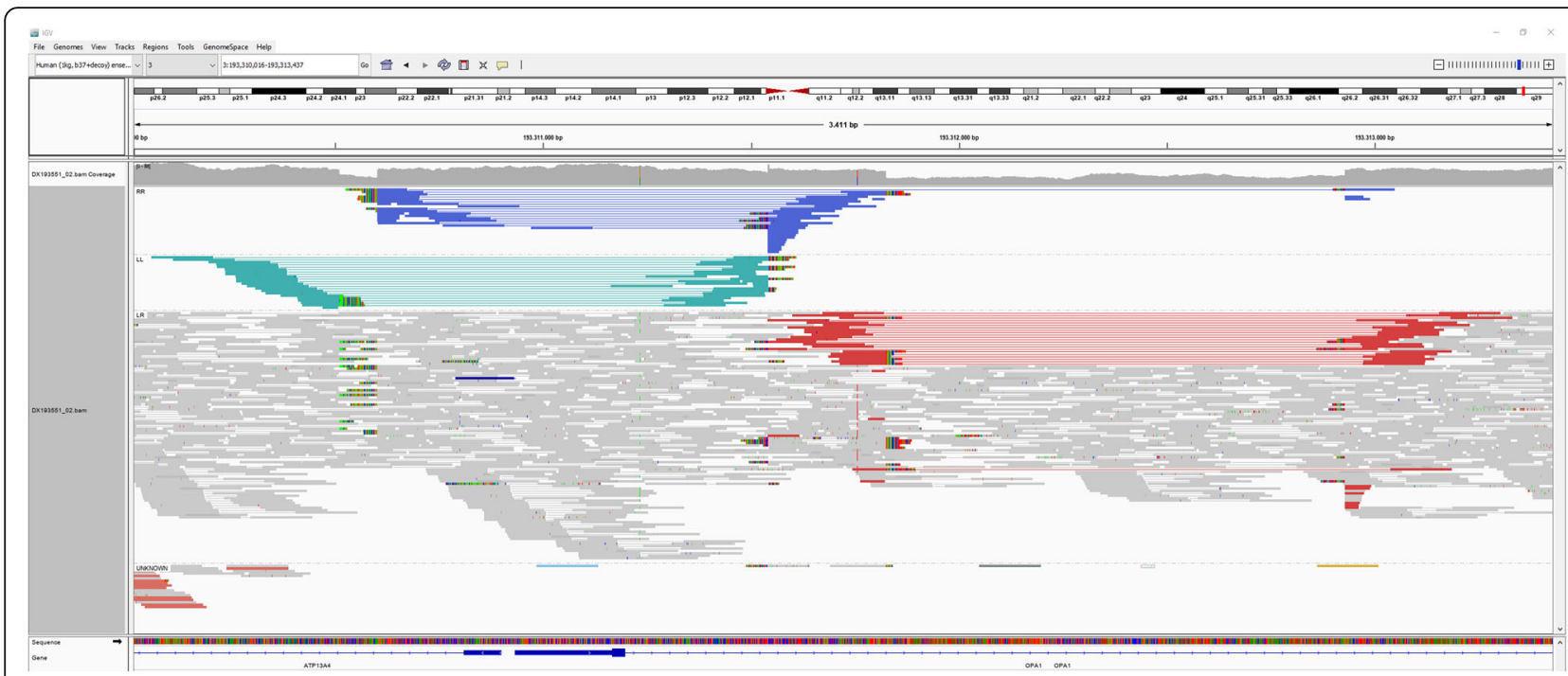

Fig. 3 Integrative Genomics Viewer (IGV) screenshot of the genomic region containing the structural variant identified in the affected proband. From top to bottom the read coverage track, the alignment track (reads colored by insert size and pair orientation and grouped by pair orientation) and the gene track are shown. Colored reads represent discordant reads with unexpected insert size and/or pair orientation when aligned to the reference genome indicating the structural variant. Blue read pairs/lines represent read pairs with right-right pair orientation, teal reads represent read pairs with left-left pair orientation and red read pairs/lines represent read pairs with aberrant insert size. Split reads with softclipped bases span the breakpoints of the structural variant

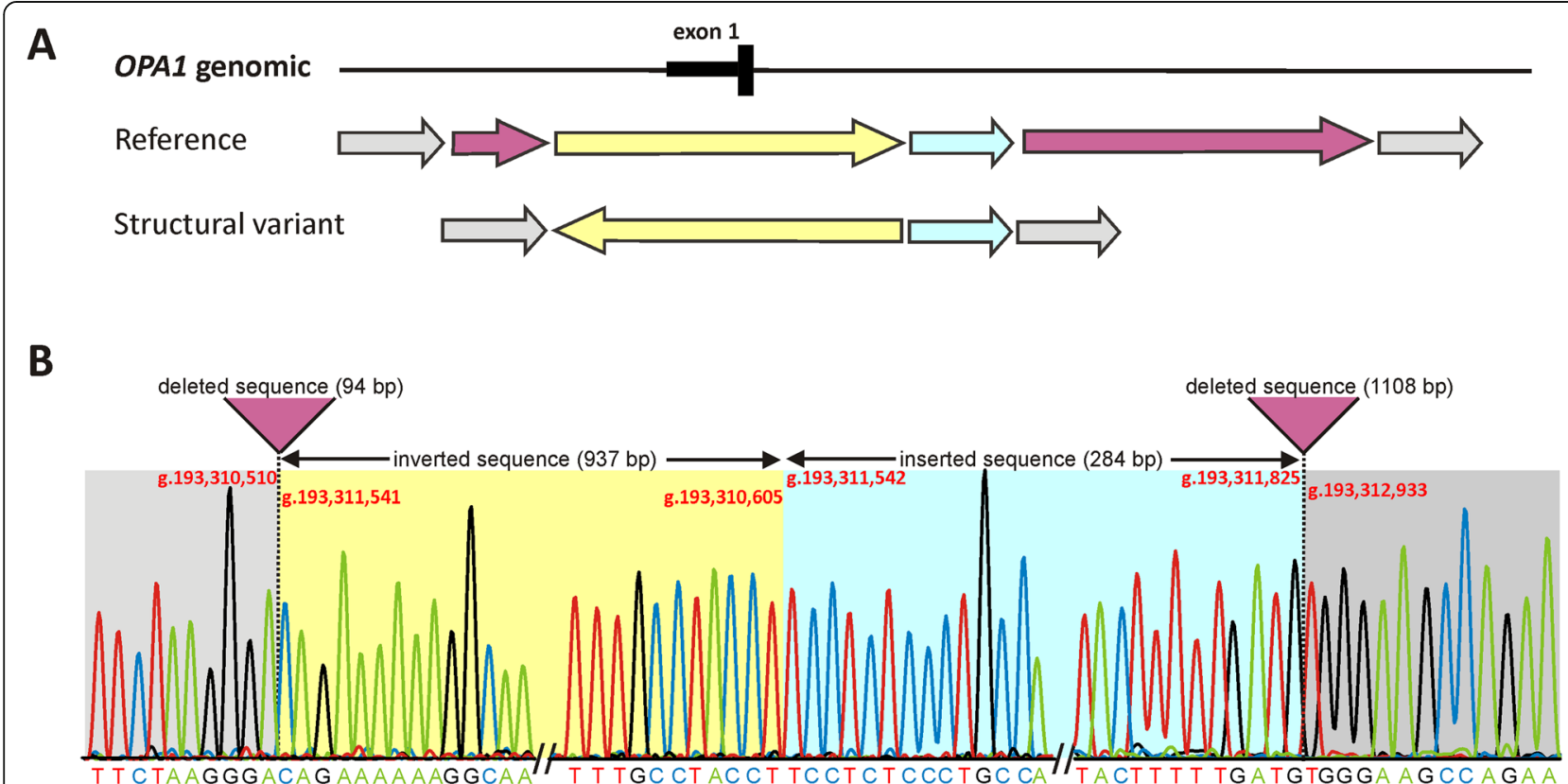

Fig. 4 Fine mapping of the structural variant by sequencing of breakpoint junctions. a Schematic representation of the structural variant. The color of the arrows indicates normal sequence (grey), inverted sequence (yellow), inserted sequence (blue) and deleted sequence (pink). b Sanger sequencing of breakpoint amplicons. The vertical dashed lines highlight the 5' and 3' breakpoints. The background colors of the electropherogram correspond to the scheme shown in (A). The deleted sequences are indicated by pink triangles. Note that the inserted sequence is actually "leftover original sequence" between the deletion and inversion events 
the guidelines of the HGVS when assigning the nomenclature to the variant. However, from a mechanistic point of view, the inserted sequence is basically "leftover original sequence" between the deletion and inversion events.

Screening for the structural variant in a cohort of 350 optic atrophy cases that had been previously tested negative for single nucleotide variants and CNVs in OPA1 was performed using patient-specific breakpoint PCRs (primer pairs OPA1-Inv-BP1-f: 5' -tgctcagcactaggcatctg-3' / OPA1Inv-BP1-r: $5^{\prime}$-ttctggacgcctctcaatct-3', OPA1-Inv-BP2-f: 5' ggaacgggaagggctaaa-3' / OPA1-Inv-BP2-r: 5'-tcgaatcaccgt ctctgaca-3', and OPA1-Inv-BP3-f: $5^{\prime}$-tgtcttcttttcttccatttccac-3' / OPA1-Inv-BP3-r: 5' -atgcttatgctctcatctgttaggg-3', respectively). All patient-specific breakpoint PCRs were performed as a duplex PCR reaction with primers that bind to the reference sequence and amplify exon 1 of OPA1 (OPA1-Ex1-F: 5' -actgagtacgggtgcctgtc-3' / OPA1-Ex1-R: $5^{\prime}$ 'gccagattagagctgcactt-3'). PCR products were resolved on a $2 \%$ agarose gel. No additional cases could be identified among the 350 unsolved cases. The variant was also absent in our internal cohort of 1400 genomes, and in the public databases gnomAD, ClinVar and DECIPHER. The lack of additional cases indicates that the variant is private, and is in line with the proposed molecular mechanism, since recurrent genomic rearrangements are most often mediated by NAHR, and not by NHEJ [25]. DNA samples were not available for familial co-segregation analysis. Hence, we could not establish whether the rearrangement was a de novo event in our proband.

Compared to single nucleotide variants and small insertions and deletions, the mapping of structural variants (SVs) is much more challenging as they cover a large portion of a read or are even larger than the read length [26]. As a consequence, the importance of SVs in Mendelian diseases is not well defined. In a recent comprehensive study, an average of 27,622 SVs was identified per human genome, including 156 inversions per genome, many of which intersected with genomic regions associated with genetic disease syndromes [27]. Whole genome sequencing detects SVs more reliably than exon-based approaches since most of the functionally relevant junctions will have both ends contained within introns or intergenic regions. Given that sequencing costs are constantly decreasing, whole genome sequencing is commissioned into routine clinical care pathways more and more frequently, offering the potential to detect SVs. Thirdgeneration sequencing technologies will probably further improve the diagnostic sensitivity as long read lengths are more likely to contain the whole SV (i.e. both breakpoints), providing less potential for error and facilitating mapping. Future studies will show whether the feasibility to detect copy-number neutral rearrangements can boost the diagnostic rate in Mendelian disorders like DOA.

\section{Supplementary Information}

The online version contains supplementary material available at https://doi. org/10.1186/s12881-020-01166-z.

Additional file 1: Figure 1. Results of perimetry.

Additional file 2: Figure 2. Results of panel D15 test.

\begin{abstract}
Abbreviations
CNV: Copy number variant; DOA: Dominant optic atrophy; FoSTeS: Fork stalling and template switching; IGV: Integrative Genomics Viewer;

LHON: Leber's hereditary optic neuropathy; NAHR: Non-allelic homologous recombination; NHEJ: Non-homologous end joining; OCT: Optical coherence tomography imaging; RNFL: Retinal nerve fiber layer; SV: Structural variant
\end{abstract}

\section{Acknowledgements}

We acknowledge support by the Deutsche Forschungsgemeinschaft and the Open Access Publishing Fund of the University of Tübingen.

\section{Authors' contributions}

$\mathrm{CK}$ and $\mathrm{FT}$ collected the clinical data. PM and TiH performed the sequencing data analysis. NW performed the breakpoint analysis and wrote the manuscript. BW and ToH supervised the study and reviewed the data. All authors read and approved the manuscript.

\section{Funding}

This study was in part funded by a grant of the Deutsche Forschungsgemeinschaft (Wi1189/11-1) as part of a joint research project TreatOPON' within the framework of the ERA-NET E-Rare 3 call. The funding body had no role in study design, data collection and analysis, decision to publish, or preparation of the manuscript. Open Access funding enabled and organized by Projekt DEAL.

\section{Availability of data and materials}

The data that support the findings of this study are available from the Institute for Medical Genetics and Genomics (IMGAG) in Tübingen but restrictions apply to the availability of these data, which were used under license for the current study, and so are not publicly available. Data are however available from the authors upon reasonable request and with permission of the IMGAG.

The datasets analysed during the current study are available at gnomAD https://gnomad.broadinstitute.org/, ClinVar (https://www.ncbi.nlm.nih.gov/), and DECIPHER (https://decipher.sanger.ac.uk/). The accession numbers used in this study and obtained from the National Center for Biotechnology Information (NCBI) include: NM_015560.2, and the NCBI37/hg19 assembly (https://www.ncbi.nlm.nih.gov/assembly/GCF_000001405.13/).

\section{Ethics approval and consent to participate}

DNA samples were recruited in accordance with the principles of the Declaration of Helsinki and were obtained with written informed consent accompanying the samples. The study was approved by the institutional review board of the Ethics Committee of the University Hospital of Tübingen (study number 637/2017BO1, dated October 23, 2017).

\section{Consent for publication}

Written informed consent to publish clinical details was obtained from the proband.

\section{Competing interests}

The authors declare that they have no competing interests.

\section{Author details}

${ }^{1}$ Institute for Ophthalmic Research, Centre for Ophthalmology, University of Tübingen, Tübingen, Germany. ${ }^{2}$ Institute of Medical Genetics and Applied Genomics, University of Tübingen, Tübingen, Germany. ${ }^{3}$ Centre for Rare Diseases, University of Tübingen, Tübingen, Germany. ${ }^{4}$ University Eye Hospital, Centre for Ophthalmology, University of Tübingen, Tübingen, Germany. 
Received: 14 August 2020 Accepted: 10 November 2020

Published online: 26 November 2020

\section{References}

1. Yu-Wai-Man P, Griffiths PG, Chinnery PF. Mitochondrial optic neuropathies disease mechanisms and therapeutic strategies. Prog Retin Eye Res. 2011;30: $81-114$

2. Jäger W. Dominant vererbte Opticusatrophie. Albrecht Von Graefes Arch Ophthalmol. 1954;155:457-84.

3. Kjer P. Hereditary infantile optic atrophy with dominant transmission. Acta Genet Stat Med. 1957:7:290-1.

4. Votruba M, Fitzke FW, Holder GE, Carter A, Bhattacharya SS, Moore AT. Clinical features in affected individuals from 21 pedigrees with dominant optic atrophy. Arch Ophthalmol. 1998;116:351-8.

5. Pineles SL, Balcer LJ. 5 Visual loss: Optic Neuropathies. In: Liu V, Galetta's, editors. Neuro-Ophthalmology, vol. 1. 3rd ed: Elsevier Inc; 2019. p. 101-96.

6. Ferré $M$, Bonneau D, Milea D, Chevrollier A, Verny C, Dollfus H, Ayuso C, Defoort S, Vignal C, Zanlonghi X, et al. Molecular screening of 980 cases of suspected hereditary optic neuropathy with a report on 77 novel OPA1 mutations. Hum Mutat. 2009:30:E692-705.

7. Almind GJ, Ek J, Rosenberg T, Eiberg H, Larsen M, LuCamp L, BrøndumNielsen K, Grønskov K. Dominant optic atrophy in Denmark-report of 15 novel mutations in OPA1, using a strategy with a detection rate of $90 \%$. BMC Med Genet. 2012;13:65.

8. Alexander C, Votruba M, Pesch UE, Thiselton DL, Mayer S, Moore A, Rodriguez M, Kellner U, Leo-Kottler B, Auburger G, et al. OPA1, encoding a dynamin-related GTPase, is mutated in autosomal dominant optic atrophy linked to chromosome 3q28. Nat Genet. 2000;26:211-5.

9. Delettre C, Lenaers G, Griffoin JM, Gigarel N, Lorenzo C, Belenguer P, Pelloquin L, Grosgeorge J, Turc-Carel C, Perret E, et al. Nuclear gene OPA1, encoding a mitochondrial dynamin-related protein, is mutated in dominant optic atrophy. Nat Genet. 2000;26:207-10

10. Westermann B. Mitochondrial fusion and fission in cell life and death. Nat Rev Mol Cell Biol. 2010;11:872-84

11. Varanita T, Soriano ME, Romanello V, Zaglia T, Quintana-Cabrera R, Semenzato M, Menabò R, Costa V, Civiletto G, Pesce P, et al. The OPA1dependent mitochondrial cristae remodeling pathway controls atrophic, apoptotic, and ischemic tissue damage. Cell Metab. 2015;21:834-44.

12. Le Roux B, Lenaers G, Zanlonghi X, Amati-Bonneau P, Chabrun F, Foulonneau T, Caignard A, Leruez S, Gohier P, Procaccio V, et al. OPA1: 516 unique variants and 831 patients registered in an updated centralized Variome database. Orphanet J Rare Dis. 2019;14:214.

13. Pesch UE, Leo-Kottler B, Mayer S, Jurklies B, Kellner U, Apfelstedt-Sylla E, Zrenner E, Alexander C, Wissinger B. OPA1 mutations in patients with autosomal dominant optic atrophy and evidence for semi-dominant inheritance. Hum Mol Genet. 2001;10:1359-68.

14. Almind GJ, Grønskov K, Milea D, Larsen M, Brøndum-Nielsen K, Ek J. Genomic deletions in OPA1 in Danish patients with autosomal dominant optic atrophy. BMC Med Genet. 2011;12:49.

15. Fuhrmann N, Alavi MV, Bitoun P, Woernle S, Auburger G, Leo-Kottler B, YuWai-Man P, Chinnery P, Wissinger B. Genomic rearrangements in OPA1 are frequent in patients with autosomal dominant optic atrophy. J Med Genet. 2009;46:136-44.

16. Bolognini R, Gerth-Kahlert C, Abegg M, Bartholdi D, Mathis N, Sturm V, Gallati S, Schaller A. Characterization of two novel intronic OPA1 mutations resulting in aberrant pre-mRNA splicing. BMC Med Genet. 2017:18:22.

17. Bonifert T, Karle KN, Tonagel F, Batra M, Wilhelm C, Theurer Y, Schoenfeld C, Kluba T, Kamenisch Y, Carelli V, et al. Pure and syndromic optic atrophy explained by deep intronic OPA1 mutations and an intralocus modifier. Brain. 2014;137(Pt 8):2164-77.

18. Weisschuh N, Sturm M, Baumann B, Audo I, Ayuso C, Bocquet B, Branham K Brooks BP, Catalá-Mora J, Giorda R, et al. Deep-intronic variants in CNGB3 cause achromatopsia by pseudoexon activation. Hum Mutat. 2020;41:255-64

19. Chen X, Schulz-Trieglaff $O$, Shaw R, Barnes B, Schlesinger F, Källberg M, Cox AJ, Kruglyak S, Saunders CT. Manta: rapid detection of structural variants and indels for germline and cancer sequencing applications. Bioinformatics. 2016:32:1220-2

20. Taschner PE, den Dunnen JT. Describing structural changes by extending HGVS sequence variation nomenclature. Hum Mutat. 2011;32:507-11.
21. Moore JK, Haber JE. Cell cycle and genetic requirements of two pathways of nonhomologous end-joining repair of double-strand breaks in Saccharomyces cerevisiae. Mol Cell Biol. 1996;16:2164-73.

22. Lupski JR. Genomic disorders: structural features of the genome can lead to DNA rearrangements and human disease traits. Trends Genet. 1998;14: 417-22.

23. Zhang F, Khajavi M, Connolly AM, Towne CF, Batish SD, Lupski JR. The DNA replication FoSTeS/MMBIR mechanism can generate genomic, genic and exonic complex rearrangements in humans. Nat Genet. 2009:41:849-53.

24. Puig M, Casillas S, Villatoro S, Cáceres M. Human inversions and their functional consequences. Brief Funct Genomics. 2015:14:369-79.

25. Liu P, Carvalho CMB, Hastings PJ, Lupski JR. Mechanisms for recurrent and complex human genomic rearrangements. Curr Opin Genet Dev. 2012;22: 211-20.

26. Sedlazeck FJ, Rescheneder P, Smolka M, Fang H, Nattestad M, von Haeseler A, Schatz MC. Accurate detection of complex structural variations using single-molecule sequencing. Nat Methods. 2018;15:461-8.

27. Chaisson MJP, Sanders AD, Zhao X, Malhotra A, Porubsky D, Rausch T, Gardner EJ, Rodriguez OL, Guo L, Collins RL, et al. Multi-platform discovery of haplotype-resolved structural variation in human genomes. Nat Commun. 2019;10:1784.

\section{Publisher's Note}

Springer Nature remains neutral with regard to jurisdictional claims in published maps and institutional affiliations.
Ready to submit your research? Choose BMC and benefit from:

- fast, convenient online submission

- thorough peer review by experienced researchers in your field

- rapid publication on acceptance

- support for research data, including large and complex data types

- gold Open Access which fosters wider collaboration and increased citations

- maximum visibility for your research: over $100 \mathrm{M}$ website views per year

At $\mathrm{BMC}$, research is always in progress.

Learn more biomedcentral.com/submissions 